\title{
Analytical description of critical dynamics for two-dimensional dissipative nonlinear maps
}

\author{
J.A. Méndez-Bermúdez ${ }^{\mathrm{a}, *}$, Juliano A. de Oliveira ${ }^{\mathrm{b}}$, Edson D. Leonel ${ }^{\mathrm{c}, \mathrm{d}}$ \\ a Instituto de Física, Benemérita Universidad Autónoma de Puebla, Apartado Postal J-48, Puebla 72570, Mexico \\ ${ }^{\mathrm{b}}$ UNESP - Univ. Estadual Paulista, Câmpus de São João da Boa Vista, Av. Professora Isette Corrêa Fontão, 505, Jardim Santa Rita das Areias, 13876-750 São João \\ da Boa Vista, SP, Brazil

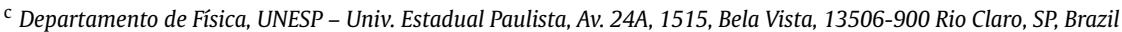 \\ d Abdus Salam International Center for Theoretical Physics, Strada Costiera 11, 34151 Trieste, Italy
}

\section{A R T I C L E I N F O}

\section{Article history:}

Received 14 January 2016

Received in revised form 3 April 2016

Accepted 4 April 2016

Available online 8 April 2016

Communicated by C.R. Doering

\section{Keywords:}

Dissipative dynamics

Nonlinear map

Scaling

\begin{abstract}
A B S T R A C T
The critical dynamics near the transition from unlimited to limited action diffusion for two families of well known dissipative nonlinear maps, namely the dissipative standard and dissipative discontinuous maps, is characterized by the use of an analytical approach. The approach is applied to explicitly obtain the average squared action as a function of the (discrete) time and the parameters controlling nonlinearity and dissipation. This allows to obtain a set of critical exponents so far obtained numerically in the literature. The theoretical predictions are verified by extensive numerical simulations. We conclude that all possible dynamical cases, independently on the map parameter values and initial conditions, collapse into the universal exponential decay of the properly normalized average squared action as a function of a normalized time. The formalism developed here can be extended to many other different types of mappings therefore making the methodology generic and robust.
\end{abstract}

(C) 2016 Elsevier B.V. All rights reserved.

\section{Introduction and model}

Phase transitions in statistical mechanics [1,2] are commonly related to abrupt changes in the spatial structure of given systems. This is related to the variation of a control parameter, hence leading to physical observables described by power laws' [3] near criticality, with well defined critical exponents [4]. In dynamical systems, the phase transitions are associated to modifications in the structure of the phase space [5], while physical observables are also characterized by critical exponents [6]. Near a phase transition, the dynamics of a system is frequently described using scaling functions [7-13] where critical exponents characterize the dynamics around criticality. In recent years, much effort has been devoted to describe the dynamics, and hence the scaling properties, of nonlinear dissipative and non-dissipative mappings [14,15]. One of the main results of those phenomenological studies is the definition of universality classes gathering maps whose average dynamics is characterized by the same critical exponents [16].

\footnotetext{
* Corresponding author. Tel.: +52 222 2295610; fax: +52 2222295611. E-mail address: jmendezb@ifuap.buap.mx (J.A. Méndez-Bermúdez). URL: http://www.ifuap.buap.mx/ jmendezb (J.A. Méndez-Bermúdez).
}

The main purpose of this work is to study the effects of dissipation on the average properties of dynamical systems. To that end we consider the dissipative dynamics produced by the following general mapping

$M_{\gamma}:\left\{\begin{array}{l}I_{n+1}=(1-\gamma) I_{n}+K f\left(\theta_{n}\right) \\ \theta_{n+1}=\theta_{n}+I_{n+1}, \quad \bmod (2 \pi),\end{array}\right.$

where $\gamma \in[0,1)$ is the dissipation parameter, $K$ controls the nonlinearity of the map, and the function $f(\theta)$ is assumed to be a periodic function with period $2 \pi$ and zero average $\langle f(\theta)\rangle=0$. Also, since a goal of this letter is to investigate the dynamics near the transition from unlimited to limited action diffusion, the range of control parameters to be considered is then $\gamma \cong 0$ and $K \gg 1$; warranting the absence of any stable periodic orbit. Notice that map $M_{\gamma}$ has the canonical form of the dissipative standard map, introduced by Zaslavskii [17] (see also [18]). However, since the function $f(\theta)$ has not been specified yet, $M_{\gamma}$ may also include dissipative discontinuous maps [6] or even dissipative polynomial maps [19] if the conditions stated above for $f(\theta)$ are relaxed. Because the determinant of the Jacobian matrix of map (1) is $1-\gamma$, $M_{\gamma}$ is area preserving only when $\gamma=0$. This fact has a very important consequence for the family of mappings we are dealing with: For $\gamma=0$ and $K \gg 1$ the action exhibits unlimited diffusion 
in phase space. However, for $\gamma \neq 0$ the phase space area contracts leading to the creation of attractors; which, given they are far from infinity, prevent unlimited action diffusion (see for example [20, 21]). This unlimited-to-limited transition in action diffusion is the transition we want to address in this letter. It is pertinent to add that even though the subject of dissipation was at the focus of seminal works on dynamical systems and chaos, see e.g. [17,18, 22-26], it is still a matter of current research [27-31].

\section{Analytical approach}

Given the symmetry of the phase space of mapping (1) (with respect to $I=0$, leading to $\left\langle I_{n}\right\rangle=0$ ), we choose to work with the squared average action by deriving its scaling properties as follows. From the first line of Eq. (1) we have that $I_{n+1}^{2}=(1-$ $\gamma)^{2} I_{n}^{2}+K^{2} f^{2}\left(\theta_{n}\right)+2(1-\gamma) K I_{n} f\left(\theta_{n}\right)$, so we can write $\left\langle I_{n+1}^{2}\right\rangle=$ $(1-\gamma)^{2}\left\langle I_{n}^{2}\right\rangle+K^{2}\left\langle f^{2}(\theta)\right\rangle+2(1-\gamma) K\left\langle I_{n}\right\rangle\langle f(\theta)\rangle$ with $\left\langle f^{2}(\theta)\right\rangle \equiv$ $\left\langle f^{2}\left(\theta_{n}\right)\right\rangle$. Since we have assumed that $\langle f(\theta)\rangle=0$, the term $2(1-$ $\gamma) K\left\langle I_{n}\right\rangle\langle f(\theta)\rangle$ can be eliminated (moreover, also $\left\langle I_{n}\right\rangle=0$ ). Therefore,

$\left\langle I_{n+1}^{2}\right\rangle=\left\langle I_{n}^{2}\right\rangle-\left(2 \gamma-\gamma^{2}\right)\left\langle I_{n}^{2}\right\rangle+K^{2}\left\langle f^{2}(\theta)\right\rangle$.

Then, by noticing that

$\left\langle I_{n+1}^{2}\right\rangle-\left\langle I_{n}^{2}\right\rangle=\frac{\left\langle I_{n+1}^{2}\right\rangle-\left\langle I_{n}^{2}\right\rangle}{(n+1)-n} \approx \frac{d J}{d n}$,

we rewrite Eq. (2) as the differential equation

$\frac{d J}{d n}=-\left(2 \gamma-\gamma^{2}\right) J+K^{2}\left\langle f^{2}(\theta)\right\rangle$,

where $J \equiv\left\langle I_{n}^{2}\right\rangle$. The approximation in Eq. (3) can be done whenever $\left\langle I_{n+1}^{2}\right\rangle-\left\langle I_{n}^{2}\right\rangle$ is sufficiently small. Note that Eq. (4) can be solved straightforwardly:

$\int_{J_{0}}^{J} \frac{d J^{\prime}}{-\left(2 \gamma-\gamma^{2}\right) J^{\prime}+K^{2}\left\langle f^{2}(\theta)\right\rangle}=\int_{n_{0}}^{n} d n^{\prime}$,

$J_{0}=\left\langle I_{0}^{2}\right\rangle=I_{0}^{2}, n_{0}=0$. Therefore, we finally write

$\left\langle I_{n}^{2}\right\rangle=I_{0}^{2} e^{-\left(2 \gamma-\gamma^{2}\right) n}+\left\langle f^{2}(\theta)\right\rangle \frac{K^{2}}{2 \gamma-\gamma^{2}}\left[1-e^{-\left(2 \gamma-\gamma^{2}\right) n}\right]$.

This result will now be discussed and fitted into several different cases.

It is relevant to stress that in the expression above the form of $f(\theta)$ is still not specified. In fact, $f(\theta)$ contributes to Eq. (6) just as the constant $\left\langle f^{2}(\theta)\right\rangle{ }^{1}$ This makes Eq. (6) very general and applicable to a large class of dissipative maps; i.e. all dissipative maps having the form (1). Then, below, to show the applicability of Eq. (6) we will consider two families of well known dissipative maps: The dissipative standard map [17] and dissipative discontinuous maps [6].

In several previous works where the scaling properties of nonlinear discontinuous maps were studied, instead of investigating $\left\langle I_{n}^{2}\right\rangle$ directly, its cumulative-normalized value

$\left\langle I_{n}^{2}\right\rangle_{\text {int }}=\frac{1}{n} \int_{n_{0}=0}^{n}\left\langle I_{n^{\prime}}^{2}\right\rangle d n^{\prime}$

\footnotetext{
1 This is particularly true when $f$ is a trigonometric function of $\theta$, which is the case in most mappings with the form (1) reported in the literature.
}
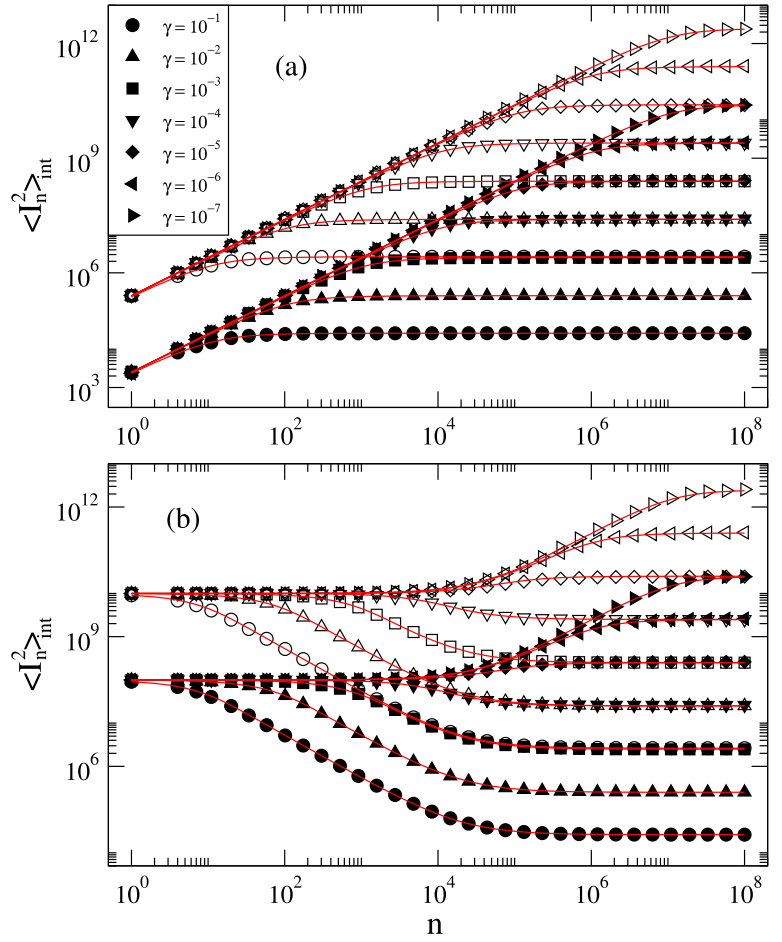

Fig. 1. (Color online) $\left\langle I_{n}^{2}\right\rangle_{\text {int }}$ as a function of $n$ for the DSM (i.e. Eq. (1) with $f(\theta)=\sin (\theta))$ when $K \gg 1$ and (a) $I_{0} \ll K$, (b) $I_{0} \gg K$. Full symbols (open symbols) correspond to $K=10^{2}\left(K=10^{3}\right)$. (a) $I_{0}=K / 100$ and (b) $I_{0}=100 K$ were used. Red full lines are Eq. (8).

was explored; see for example [14]. Fortunately, from Eq. (6) we can also write down an explicit expression for $\left\langle I_{n}^{2}\right\rangle_{\text {int }}$ :

$$
\begin{aligned}
\left\langle I_{n}^{2}\right\rangle_{\text {int }} & =\frac{I_{0}^{2}}{n\left(2 \gamma-\gamma^{2}\right)}\left[1-e^{-\left(2 \gamma-\gamma^{2}\right) n}\right] \\
& +\left\langle f^{2}(\theta)\right\rangle \frac{K^{2}}{n\left(2 \gamma-\gamma^{2}\right)}\left[n-\frac{1-e^{-\left(2 \gamma-\gamma^{2}\right) n}}{2 \gamma-\gamma^{2}}\right] .
\end{aligned}
$$

Thus, below we numerically compute $\left\langle I_{n}^{2}\right\rangle_{\text {int }}$ for map (1) following two steps: First we calculate the cumulative average squared action over the orbit associated with the initial condition $j$ as $\left\langle I_{n, j}^{2}\right\rangle=(n+1)^{-1} \sum_{i=0}^{n} I_{i, j}^{2}$, where $i$ refers to the $i$-th iteration of the map. Then, we average over $M$ independent realizations of the map (by randomly choosing values of $\theta_{0}$ ) to finally obtain $\left\langle I_{n}^{2}\right\rangle_{\text {int }} \equiv M^{-1} \sum_{j=1}^{M}\left\langle I_{n, j}^{2}\right\rangle$. In our simulations, for each combination of parameters $\left(I_{0}, \gamma, K\right)$, we consider an ensemble of $10^{3}$ initial random phases uniformly distributed in the interval $0<\theta_{0}<2 \pi$. We have verified that larger ensembles lead to qualitatively the same results.

\section{Discussion}

Let us now describe the behavior of $\left\langle I_{n}^{2}\right\rangle_{\text {int }}$ obtained from the numerical iteration of map (1). In Fig. 1 we present $\left\langle I_{n}^{2}\right\rangle_{\text {int }}$ as a function of $n$ for the dissipative standard map (DSM), which is defined by Eq. (1) with $f(\theta)=\sin (\theta)$ [17]; thus $\left\langle f^{2}(\theta)\right\rangle=1 / 2$. We have considered $I_{0} \ll K$ and $I_{0} \gg K$ separately (Figs. 1(a) and 1(b), respectively) since the behavior of $\left\langle I_{n}^{2}\right\rangle_{\text {int }}$ vs. $n$ shows important differences in both cases: For $I_{0} \ll K$ there are two regimes separated by the crossover iteration number $n_{\mathrm{CO}}$; a growth regime for $n<n_{\mathrm{CO}}$ and the saturation regime where $\left\langle I_{n}^{2}\right\rangle_{\text {int }} \approx I_{\text {sat }}^{2}$ for $n>n_{\mathrm{CO}}$. This is a typical scenario observed in phase transitions [5]; in this case a transition from diffusion to saturation. From Fig. 1(a) it 


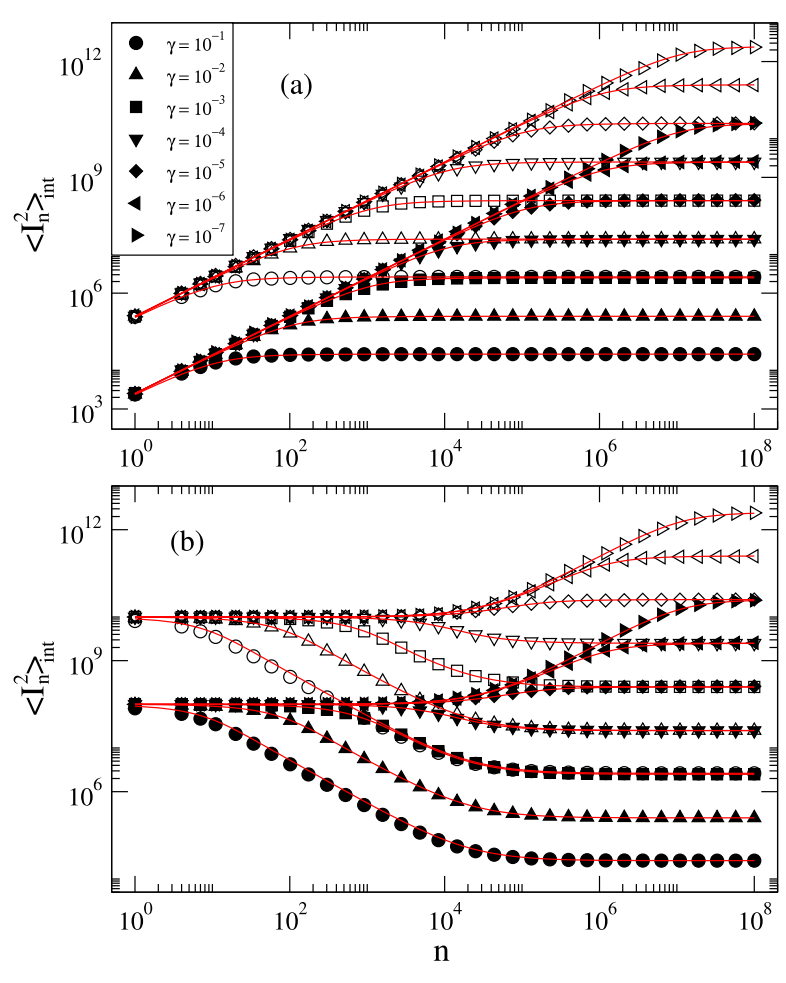

Fig. 2. (Color online) Same as in Fig. 1 for the DDM, i.e. Eq. (1) with $f(\theta)=$ $\sin (\theta) \operatorname{sgn}[\cos (\theta)]$.

can be observed that the nonlinearity parameter $K$ displaces the curves $\left\langle I_{n}^{2}\right\rangle_{\text {int }}$ vs. $n$ vertically ${ }^{2}$ while the dissipation parameter $\gamma$ determines $n_{\mathrm{CO}}$. When $I_{0} \gg K$ the situation is more elaborate, see Fig. 1(b). For small $n$ the curves $\left\langle I_{n}^{2}\right\rangle_{\text {int }}$ vs. $n$ are approximately constant and equal to $I_{0}^{2} ;{ }^{3}$ but there is a critical value for $\gamma$ such that if $\gamma<\gamma_{\mathrm{cr}}$ or $\gamma>\gamma_{\mathrm{cr}},\left\langle I_{n}^{2}\right\rangle_{\text {int }}$ increases or decreases, respectively, as a function of $n$ before saturating at $n \rightarrow \infty$.

It is important to remark that in both cases, $I_{0} \ll K$ and $I_{0} \gg K$, Eq. (8) describes remarkably well the behavior of $\left\langle I_{n}^{2}\right\rangle_{\text {int }}$; see red full lines in Fig. 1. Actually, this excellent correspondence will allow us to derive close expressions for $n_{\mathrm{CO}}, I_{\text {sat }}^{2}$, and $\gamma_{\mathrm{cr}}$ below.

For the dissipative discontinuous map (DDM), the function $f(\theta)$ in Eq. (1) is chosen as $f(\theta)=\sin (\theta) \operatorname{sgn}[\cos (\theta)]$ [6]. Note that, as well as for the DSM, here $\left\langle f^{2}(\theta)\right\rangle=1 / 2$. This means that (even though the corresponding non-dissipative mappings represent qualitatively different dynamical systems) according to Eq. (8) we expect no difference in the behavior of $\left\langle I_{n}^{2}\right\rangle_{\text {int }}$ for both families of dissipative maps. This fact is evidenced in Fig. 2 where we show $\left\langle I_{n}^{2}\right\rangle_{\text {int }}$ as a function of $n$ for the DDM. Indeed, since we used the same parameter values as in Fig. 1, it is not possible to detect any difference (by eye) between the curves of Figs. 1 and 2 corresponding to the DSM and the DDM, respectively. Therefore, below we do not treat DSM and DDM independently anymore.

In the following we describe different relevant behaviors of $\left\langle I_{n}^{2}\right\rangle$ predicted by Eq. (6) in connection with previous results from scaling studies that our approach accurately validates.

\footnotetext{
2 This behavior is already anticipated by the second part of Eq. (8) as $\left\langle I_{n}^{2}\right\rangle_{\text {int }} \propto K^{2}$. Indeed, it can also be directly observed from the first equation of map (1). Recall that $I_{0} \ll K$ here.

${ }^{3}\left\langle I_{n}^{2}\right\rangle_{\text {int }} \approx I_{0}^{2}$ can be obtained by taking the limit $n \rightarrow 0$ on Eq. (8). Moreover, it can also be observed from the first equation of map (1). Recall that $I_{0} \gg K$ here.
}

\subsection{Large-time limit: saturation of $\left\langle I_{n}^{2}\right\rangle$}

For any $\gamma>0$ the saturation of $\left\langle I_{n}^{2}\right\rangle$ is observed in the limit of $n \rightarrow \infty$ for the DSM and the DDM; see Figs. 1 and 2 where $\left\langle I_{n}^{2}\right\rangle_{\text {int }} \approx$ const $=I_{\text {sat }}^{2}$ for large $n$. In addition, the saturation of $\left\langle I_{n}^{2}\right\rangle$, and related quantities, have also been reported for many other dissipative maps; see e.g. [9,32,33]. By taking this limit into Eq. (6) we get

$I_{s a t}^{2} \equiv \lim _{n \rightarrow \infty}\left\langle I_{n}^{2}\right\rangle=\left\langle f^{2}(\theta)\right\rangle \frac{K^{2}}{2 \gamma-\gamma^{2}}$.

This provides $I_{\text {sat }}^{2} \propto K^{2} \gamma^{-1}$, when $\gamma \ll 1$, as reported in [9,34,35] for several dissipative maps.

\subsection{Small initial action $I_{0} \ll K$}

When $I_{0} \ll K$, case depicted in Figs. 1(a) and 2(a), the behavior of $\left\langle I_{n}^{2}\right\rangle$ as a function of $n$ is quite simple: There are two regimes separated by the crossover iteration number $n_{\mathrm{CO}}$; a growth regime for $n<n_{\mathrm{CO}}$ and the saturation regime for $n>n_{\mathrm{CO}}$. As a matter of fact, from Eq. (6) this situation can be well described by

$\left\langle I_{n}^{2}\right\rangle \approx I_{\text {sat }}^{2}\left[1-e^{-\left(2 \gamma-\gamma^{2}\right) n}\right]$,

which provides the growth $\left\langle I_{n}^{2}\right\rangle \approx\left\langle f^{2}(\theta)\right\rangle K^{2} n$ for small $n$, similar to normal diffusion, and the saturation $\left\langle I_{n}^{2}\right\rangle \approx I_{\text {sat }}^{2}$ for large $n$. Moreover, from Eq. (10) it is clear that the ratio $\left\langle I_{n}^{2}\right\rangle / I_{\text {sat }}^{2}$ is a universal function of the variable $\bar{n} \equiv n / n_{\mathrm{CO}}$ :

$\frac{\left\langle I_{n}^{2}\right\rangle}{I_{\text {sat }}^{2}} \approx 1-e^{-\left(2 \gamma-\gamma^{2}\right) n}=1-e^{-\bar{n}}$,

where the crossover iteration number, that does not depend on $K$ nor on $I_{0}$, is naturally defined as

$n_{\mathrm{CO}} \equiv \frac{1}{2 \gamma-\gamma^{2}}$.

In fact, $n_{\text {CO }}$ was correctly reported to be proportional to $\gamma^{-1}$ in $[6,9,11,12,15,32,34,35]$, when $\gamma \ll 1$, for several dissipative maps.

Notice that by expanding the exponential in Eq. (11) one can also obtain the scaling function

$\frac{\left\langle I_{n}^{2}\right\rangle}{I_{\text {sat }}^{2}} \approx \frac{\bar{n}}{1+\bar{n}}$,

which was heuristically proposed in [11] for the DSM and validated, indirectly, for the dissipative versions of the oval billiard [36], the Lorentz gas [12], and the elliptical billiard [15].

\subsection{Large initial action $I_{0} \gg K$}

Given the definition of $I_{\text {sat }}^{2}$, Eq. (9), we can rewrite Eq. (6) as

$\left\langle I_{n}^{2}\right\rangle=\left(I_{0}^{2}-I_{\text {sat }}^{2}\right) e^{-\left(2 \gamma-\gamma^{2}\right) n}+I_{\text {sat }}^{2}$,

that can be well approximated by

$\left\langle I_{n}^{2}\right\rangle \approx I_{0}^{2} e^{-\left(2 \gamma-\gamma^{2}\right) n}+I_{\text {sat }}^{2}$,

when $I_{0} \gg K$ and $\gamma>\gamma_{\mathrm{cr}}$. Equation (15) predicts the exponential decay of $\left\langle I_{n}^{2}\right\rangle$ for dissipative maps before saturation, as already illustrated in Figs. 1(b) and 2(b). This exponential decay of action (and related quantities) has also been reported for dissipative maps in $[6,32,35]$. Moreover, from Eq. (15) we can recognize the following universal function of the variable $\bar{n}$ : 


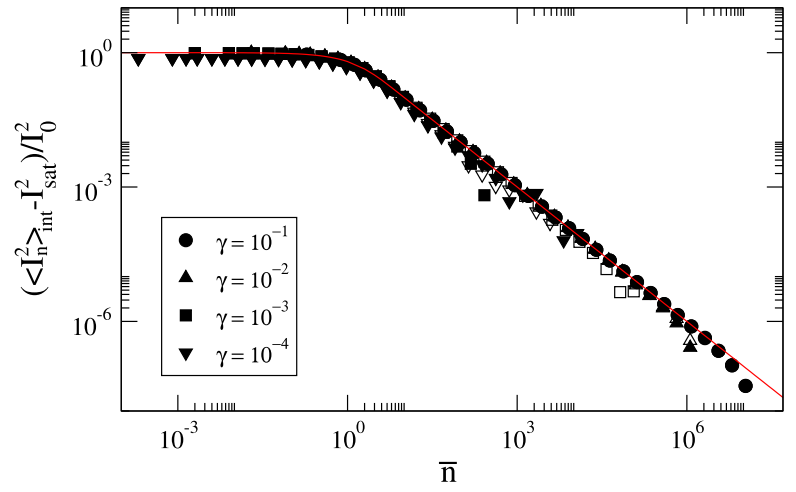

Fig. 3. (Color online) $\left(\left\langle I_{n}^{2}\right\rangle_{\text {int }}-I_{\text {sat }}^{2}\right) / I_{0}^{2}$ as a function of $\bar{n}=n / n_{\mathrm{CO}}$ for the DSM when $I_{0} \gg K$ and $\gamma>\gamma_{\mathrm{cr}} \approx 2.5 \times 10^{-5}$, see Eq. (19). Full symbols (open symbols) correspond to $K=10^{2}\left(K=10^{3}\right)$. $I_{0}=100 K$ was used. Red full line is Eq. (17).

$\frac{\left\langle I_{n}^{2}\right\rangle-I_{\text {sat }}^{2}}{I_{0}^{2}} \approx e^{-\left(2 \gamma-\gamma^{2}\right) n}=e^{-\bar{n}}$.

Similarly, for $\left\langle I_{n}^{2}\right\rangle_{\text {int }}$ we can write

$\frac{\left\langle I_{n}^{2}\right\rangle_{\text {int }}-I_{\text {sat }}^{2}}{I_{0}^{2}} \approx \frac{1-e^{-\bar{n}}}{\bar{n}}$.

Then, in Fig. 3 we show $\left(\left\langle I_{n}^{2}\right\rangle_{\text {int }}-I_{\text {sat }}^{2}\right) / I_{0}^{2}$ as a function of $\bar{n}$ for the DSM. It is clear that all numerically obtained curves (symbols) collapse on top of Eq. (17) (red full line). However, due to oscillations of $\left\langle I_{n}^{2}\right\rangle_{\text {int }}$ around $I_{\text {sat }}^{2}$ when $n>n_{\mathrm{CO}}$, enhanced here by the computation of the difference $\left\langle I_{n}^{2}\right\rangle_{\text {int }}-I_{\text {sat }}^{2}$, in Fig. 3 we observe symbols that deviate from a perfect exponential decay.

\subsection{Equilibrium condition: $\left\langle I_{n}^{2}\right\rangle=$ const}

We have shown above that Eq. (6) reproduces behaviors previously reported for $\left\langle I_{n}^{2}\right\rangle$ and $\left\langle I_{n}^{2}\right\rangle_{\text {int }}$. Moreover, in addition, we can predict the following unexpected situation: By equating Eqs. (6) and (9) we assume that $\left\langle I_{n}^{2}\right\rangle$ remains constant at all times $n$, if the appropriate initial action $I_{0}$ is chosen. Indeed, such $I_{0}$ reads

$I_{0}^{2}=\left\langle f^{2}(\theta)\right\rangle \frac{K^{2}}{2 \gamma-\gamma^{2}}=I_{\text {sat }}^{2}$.

With this choice of $I_{0}$, dissipation and diffusion compensate each other exactly and the squared average action does not increase nor decrease; i.e. it remains constant and equal to $I_{0}^{2}$ (or $I_{\text {sat }}^{2}$ ). In Fig. 4 we show $\left\langle I_{n}^{2}\right\rangle$ (which in this case coincides with $\left\langle I_{n}^{2}\right\rangle_{\text {int }}$ ) as a function of $n$ for the DSM and the DDM for several combinations of $K$ and $\gamma$ (symbols). However, since we have used as initial action the value of $I_{0}$ given by Eq. (18), the curves $\left\langle I_{n}^{2}\right\rangle$ vs. $n$ are straight horizontal lines equal to $I_{0}^{2}$ (red full lines).

Finally, we recall from Figs. 1(b) and 2(b) that there is a critical value of $\gamma$ such that if $\gamma<\gamma_{\text {cr }}$ or $\gamma>\gamma_{\text {cr }}$ then $\left\langle I_{n}^{2}\right\rangle_{\text {int }}$ increases or decreases, respectively, as a function of $n$ before saturating at $n \rightarrow \infty$. In fact, note from Eq. (18) that conditions $\gamma<\gamma_{\mathrm{cr}}$ and $\gamma>\gamma_{\text {cr }}$ translate into $I_{0}^{2}<I_{\text {sat }}^{2}$ and $I_{0}^{2}>I_{\text {sat }}^{2}$, respectively; therefore we obtain

$\gamma_{\mathrm{cr}}=1-\sqrt{1-\left\langle f^{2}(\theta)\right\rangle \frac{K^{2}}{I_{0}^{2}}}$.

Equation (19) thus provides $\gamma_{\mathrm{cr}} \approx 2.5 \times 10^{-5}$ for the parameters used in Figs. 1(b), 2(b), and 3.

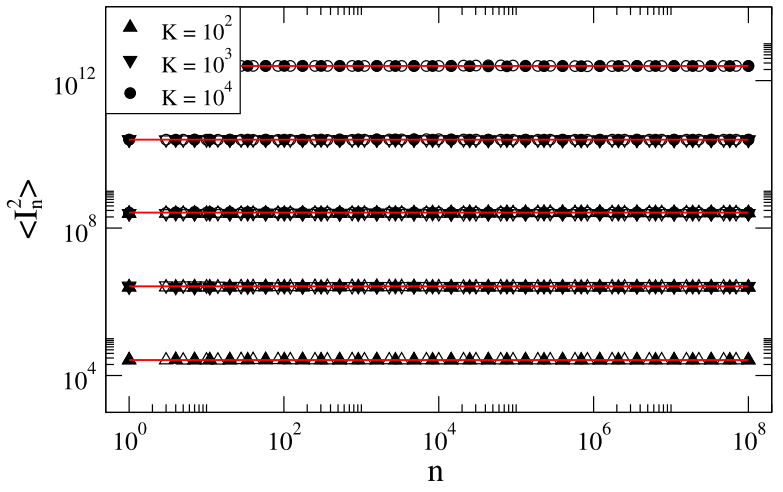

Fig. 4. (Color online) $\left\langle I_{n}^{2}\right\rangle=\left\langle I_{n}^{2}\right\rangle_{\text {int }}$ as a function of $n$ for the DSM (full symbols) and the DDM (open symbols). For each value of $K$ we used three values of $\gamma: 10^{-1}$, $10^{-2}$, and $10^{-3} \cdot I_{0}$ is given by Eq. (18). Red full lines are also Eq. (18).

\section{Summary and conclusions}

We developed a transparent and powerful analytical approach able to accurately describe the behavior of the average squared action of dissipative nonlinear mappings near the transition from unlimited to limited action diffusion, for $\gamma \cong 0$, as a function of the discrete time and the parameters controlling nonlinearity and dissipation, see Eq. (6). We also described in detail cases previously discussed in the literature that our approach accurately validates. We shown that from our Eq. (6) it is possible to derive universal scaling functions that were obtained before only approximately; see Eqs. (9), (12), (13), and (17). Moreover, in Eq. (18), we were able to state the unforeseen condition for which dissipation and diffusion compensate each other exactly keeping constant the average squared action.

Furthermore from the rewritten Eq. (6), i.e. Eq. (14), it is possible to identify the universal scaling function

$\overline{\left\langle I_{n}^{2}\right\rangle}=e^{-\bar{n}}$

that embraces all behaviors discussed above. Here

$\overline{\left\langle I_{n}^{2}\right\rangle} \equiv \frac{\left\langle I_{n}^{2}\right\rangle-I_{\text {sat }}^{2}}{I_{0}^{2}-I_{\text {sat }}^{2}}, \quad \bar{n} \equiv\left(2 \gamma-\gamma^{2}\right) n$,

and $I_{\text {sat }}^{2}$ given by Eq. (9). Equation (20) explicitly shows that the net effect of dissipation is indeed the exponential decay of the (properly normalized) average squared action.

We would like to point out that our predictions may also be applicable to variations of the DSM that have been considered in the literature as well as to other systems described by mappings, such as Fermi-Ulam and bouncer maps, where dissipation appears either as drag forces or inelastic collisions.

Finally, we stress that the non-dissipative case, i.e. $\gamma=0$ in mapping (1), can also be investigated by the use of the approach developed here. However, since the main focus of the paper is on the dissipative case, in order to do not distract the reader, we left the discussion of this case to the Appendix.

\section{Acknowledgements}

J.A.M.-B. thanks to FAPESP (Grants No. 2013/14655-9 and No. 2014/25997-0), CONACYT (Grants No. I0010-2014-246246 and No. CB-2013/220624), and VIEP-BUAP (Grant No. MEBJ-EXC15-I) for financial support. J.A.O. acknowledges financial support from PROPe/UNESP, CNPq (Grant No. 311105/2015-7), and FAPESP (Grant No. 2014/18672-8). E.D.L. acknowledges support from CNPq (Grant No. 303707/2015-1), FAPESP (Grant No. 2012/23688-5) and FUNDUNESP, Brazilian agencies. 


\section{Appendix A. Non-dissipative case: $\gamma=0$}

Indeed, the case of no dissipation can also be taken into account using our approach by setting $\gamma=0$ into Eq. (4), leading to

$\left\langle I_{n}^{2}\right\rangle=I_{0}^{2}+\left\langle f^{2}(\theta)\right\rangle K^{2} n$.

Notice that Eq. (22) reproduces the scaling laws

$\left\langle I_{n}^{2}\right\rangle\left\{\begin{array}{ccc}\propto K^{2} n, & \text { when } & I_{0} \ll K, \\ \approx I_{0}^{2}, & n<n_{\mathrm{CO}} \\ \propto K^{2} n, & n>n_{\mathrm{CO}}\end{array}\right\} \quad$ when $I_{0} \gg K$,

reported in [10] and [37] for the standard map and non-dissipative discontinuous maps, respectively. In (23), $n_{\mathrm{CO}}$ is a crossover iteration number that separates the regime of constant action and the regime of Fermi acceleration characterized by $\left\langle I_{n}^{2}\right\rangle \propto K^{2} n$. Moreover, it is clear from Eq. (22) that the ratio $\left\langle I_{n}^{2}\right\rangle / I_{0}^{2}$ is a universal function of the variable $\bar{n}=n / n_{\mathrm{CO}}$ :

$\frac{\left\langle I_{n}^{2}\right\rangle}{I_{0}^{2}}=1+\left\langle f^{2}(\theta)\right\rangle \frac{K^{2}}{I_{0}^{2}} n=1+\bar{n}$,

where $n_{\mathrm{CO}}$ is naturally defined as

$n_{\mathrm{CO}} \equiv \frac{I_{0}^{2}}{\left\langle f^{2}(\theta)\right\rangle K^{2}}$.

It is relevant to recall that, by the use of scaling arguments, the universality of the ratio $\left\langle I_{n}^{2}\right\rangle / I_{0}^{2}$ was deduced in [10,12,37-39]; there, $n_{\mathrm{CO}}$ was also reported to be proportional to $I_{0}^{2} K^{-2}$, in agreement with Eq. (25).

Furthermore, scalings (23) have also been validated for several dynamical systems which are represented locally (but not exclusively) by the standard map such as the Fermi-Ulam model $[5,7$, $8,13,16,40,41]$, time-dependent potential wells [42,43], and waveguide billiards [16,44]; among others [14,15,45]. Also, notice that the non-dissipative discontinuous map $[37,46]$ has been shown to describe systems including 2D billiard models, like the stadium billiard [47,48] and polygonal billiards [49,50]. Thus, our results for the non-dissipative case [i.e. Eqs. (22), (24) and (25)] are not restricted to the standard map nor to the non-dissipative discontinuous map.

\section{References}

[1] A.M. Figueiredo-Neto, S.R.A. Salinas, The Physics of Lyotropic Liquid Crystals: Phase Transitions and Structural Properties, Monographs on the Physics and Chemistry of Materials, Oxford University Press, New York, 2005.

[2] L.P. Kadanoff, Statistical Physics: Statics, Dynamics and Renormalization, World Scientific Publishing, Singapore, 2000.
[3] W.D. McComb, Renormalization Methods: A Guide for Beginners, Oxford University Press, Oxford, 2004

[4] F. Reif, Fundamentals of Statistical and Thermal Physics, McGraw-Hill, New York, 1965.

[5] E.D. Leonel, P.V.E. McClintock, J.K.L. da Silva, Phys. Rev. Lett. 93 (2004) 14101.

[6] R. Aguilar-Sanchez, E.D. Leonel, J.A. Mendez-Bermudez, Phys. Lett. A 377 (2013) 3216.

[7] O.F.A. Bonfim, Phys. Rev. E 79 (2009) 056212.

[8] O.F.A. Bonfim, Int. J. Bifurc. Chaos 22 (2012) 1250140.

[9] A.L.P. Livorati, D.G. Ladeira, E.D. Leonel, Phys. Rev. E 78 (2008) 056205.

[10] D.G. Ladeira, J.K.L. Silva, J. Phys. A, Math. Theor. 40 (2007) 11467.

[11] D.F.M. Oliveira, M. Robnik, E.D. Leonel, Phys. Lett. A 376 (2012) 723.

[12] D.F.M. Oliveira, J. Vollmer, E.D. Leonel, Physica D 240 (2011) 389.

[13] D.G. Ladeira, J.K.L. da Silva, Phys. Rev. E 73 (2006) 026201.

[14] D.G. Ladeira, J.K.L. da Silva, J. Phys. A, Math. Theor. 41 (2008) 365101.

[15] D.F.M. Oliveira, M. Robnik, Phys. Rev. E 83 (2011) 026202.

[16] E.D. Leonel, Math. Probl. Eng. 2009 (2009) 367921

[17] G.M. Zaslavskii, Phys. Lett. A 69 (1978) 145.

[18] O.F. Vlasova, G.M. Zaslavsky, Phys. Lett. A 105 (1984) 1.

[19] B.V. Chirikov, F.M. Izrailev, Physica D 2 (1981) 30.

[20] A.J. Lichtenberg, M.A. Lieberman, Regular and Chaotic Dynamics, SpringerVerlag, New York, 1992.

[21] H.G. Schuster, W. Just, Deterministic Chaos: An Introduction, fourth edition, Wiley-VCH Verlag, New York, 2005.

[22] F.M. Izrailev, M.I. Rabinovich, A.D. Ugodnikov, Phys. Lett. A 86 (1981) 321.

[23] R.V. Jensen, C.R. Oberman, Physica D 4 (1982) 183.

[24] K.Y. Tsang, M.A. Lieberman, Phys. Lett. A 103 (1984) 175; K.Y. Tsang, M.A. Lieberman, Physica D 11 (1984) 147; K.Y. Tsang, M.A. Lieberman, Physica D 21 (1986) 401

[25] P. Grassberger, I. Procaccia, Physica D 9 (1983) 189; P. Grassberger, I. Procaccia, Physica D 13 (1984) 24.

[26] O.F. Vlasova, G.M. Zaslavsky, Phys. Lett. A 99 (1983) 405.

[27] C.S. Rodrigues, A.V. Chechkin, A.P.S. de Moura, C. Grebogi, R. Klages, Europhys. Lett. 108 (2014) 40002.

[28] Y. Joshi, D. Blackmore, Chaos Solitons Fractals 68 (2014) 123.

[29] M. Rack, K.H. Spatschek, A. Wingen, Chaos 22 (2012) 123114.

[30] L. Renna, F. Paladini, Theor. Math. Phys. 168 (2011) 1009.

[31] W. Ott, M. Stenlund, Commun. Math. Phys. 296 (2010) 215.

[32] E.D. Leonel, L.A. Bunimovich, Phys. Rev. E 82 (2010) 016202.

[33] D.F.M. Oliveira, E.D. Leonel, M. Robnik, Phys. Lett. A 375 (2011) 3365.

[34] E.D. Leonel, A.L.P. Livorati, Physica A 387 (2008) 1155.

[35] J.A. de Oliveira, E.D. Leonel, J. Phys. A, Math. Theor. 45 (2012) 165101.

[36] D.F.M. Oliveira, E.D. Leonel, Physica A 389 (2010) 1009.

[37] J.A. Mendez-Bermudez, R. Aguilar-Sanchez, Phys. Rev. E 85 (2012) 056212.

[38] D.G. Ladeira, E.D. Leonel, Phys. Rev. E 81 (2010) 036216.

[39] D.F.M. Oliveira, E.D. Leonel, Chaos 22 (2012) 026123.

[40] J.K.L. da Silva, D.G. Ladeira, E.D. Leonel, P.V.E. McClintock, Braz. J. Phys. 36 (2006) 700.

[41] E.D. Leonel, J.K.L. da Silva, S.O. Kamphorst, Physica A 331 (2004) 435

[42] E.D. Leonel, P.V.E. McClintock, Chaos 15 (2005) 33701.

[43] E.D. Leonel, P.V.E. McClintock, J. Phys. A, Math. Gen. 37 (2004) 8949.

[44] E.D. Leonel, Phys. Rev. Lett. 98 (2007) 114102.

[45] J.A. de Oliveira, R.A. Bizão, E.D. Leonel, Phys. Rev. E 81 (2010) 046212.

[46] F. Borgonovi, Phys. Rev. Lett. 80 (1998) 4653.

[47] F. Borgonovi, G. Casati, B. Li, Phys. Rev. Lett. 77 (1996) 4744.

[48] G. Casati, T. Prosen, Phys. Rev. E 59 (1999) R2516.

[49] G. Casati, T. Prosen, Phys. Rev. Lett. 85 (2000) 4261.

[50] T. Prosen, M. Znidaric, Phys. Rev. Lett. 87 (2001) 114101. 